\title{
Familiarising Probabilistic Distance Clustering System of Evolving Awale Player
}

\author{
Randle Oluwarotimi Abayomi ${ }^{1}$ and Keneilwe $\mathrm{Zuva}^{2}$ \\ ${ }^{1}$ Department of Computer Science, Tshwane University of Technology, South Africa \\ rotran86@yahoo.com \\ ${ }^{2}$ Department of Computer Science, University of Botswana \\ mapokak@mopipi.ub. bw
}

\begin{abstract}
This study developed a new technique based on Probabilistic Distance Clustering (PDC) for evolving Awale player and to compare its performance with that of a technique based on approximation of minimum and maximum operators with generalized mean-value operator. The basic theory of pd-clustering is based on the assumption that the probability of an Euclidean point belonging to a cluster is inversely proportional to its distance from the cluster centroid. Treating game strategies as a vector space model, it is possible to extend pd-clustering technique to game playing by estimating the probability that a given strategy is in a certain cluster of game strategies. As a result, the strategy that has the highest probability and shortest distance to a cluster of alternative strategies is recommended for the player.
\end{abstract}

\section{KEYWORDS}

Minimax Search, Mahalanobis Distance, Hybrid, Awale

\section{INTRODUCTION}

As we know, game problem solving generally has Non-Polynomial (NP) time computational complexity and as such, there is no algorithm in existence that can solve game problems in polynomial time. The general solution to solving game problem is based on searching and there are two types of search approaches, exhaustive and heuristic. While exhaustive search usually employs depth-first or breath-first traversal algorithm to produce the entire state space for the problem being considered, heuristic search such as alpha-beta pruning generates an approximate solution that can either be a point in the state space or a path from the initial state.

In reality, exhaustive search is not feasible as state space can be large to subdue the computational power of a single processor Von Neumann computer. Heuristic search techniques such as minimax algorithm and its variants such as alpha-beta pruning have been applied to two-person zero-sum board game such as Chess, Poker, Bagamonn and Awale. These techniques limit search to certain subspaces of the entire state space to obtain appropriate game solutions. However, the main challenge in developing minimax search algorithms for game playing is how to construct an effective evaluation function. The design of an evaluation function requires the game designer to have considerable knowledge of the game. Based on this knowledge, the designer can then create a set of playing principles that are embedded into the evaluation function. But, it has been long 
International journal on applications of graph theory in wireless ad hoc networks and sensor networks

(GRAPH-HOC) Vol.4, No.2/3, September 2012

concluded that to improve game strength of Awale, it is important to have a larger endgame database than to have a better evaluation function (Allis et al. 1994; Lincke \& Marzette 2000).

As we also know, game playing is one area that machine intelligence applications have been demonstrated to be useful, but developing intelligence computer game programs that can outplay human ingenuity is still a difficult task. Recently, some researchers in game playing (Donkers et al., 2002; Davis and Kendell, 2002; Dauod et al., 2004; Olugbara, et al., 2006; Olugbara, et al. 2007; Akinyemi et al., 2008; Akinyemi et al., 2009) are looking at machine intelligence techniques such as opponent model, evolutionary programming, genetic algorithm, casebase reasoning and nearest neighbour search algorithm to help design Awale game programs. However, for most games, it is difficult to create a large enough set of game patterns database (Agbinya, 2004). This study attempts to develop a new machine intelligence game playing technique based on pd-clustering to evolve an Awale player. A fictitious endgame strategies database will be developed and used to train the player to reasonably play Awale.

Awale is a two-person-zero-sum strategic board game that belongs to the family of Mancala games and it is known by several names including, Ayo, Awari and Owari, to name a few. It is a seed sowing game that involves two players North (Min) and South (Max) with adiabatically opposing views. The game has 12 holes or pits and it is of size 12 with 6 holes on North and South sides. There are 48 seeds in Awale with 4 seeds sowed in each hole at the commencement of the game and 2 extra holes to serve as seed bags where the captured seeds are stored. Each player sows seeds on the holes from right to left that is counter-clockwisely thereby increasing the number of seeds in holes, but making the starting hole empty. In this game, an empty hole has zero seeds, a hole containing 12-17 seeds is called odu or kroo and it can capture up to 15 seeds at a time.

The current player selects all seeds from a non-empty hole and sows them counter- clockwisely into corresponding holes excluding the starting hole. If the last seed is sown into a hole of the opponent, which has 2 or 3 seeds, the player captures all the consecutive 2 or 3 seeds and keeps them in his bag. This capturing phenomenon is called 2-3 capturing rule and this rule varies for different variants of Mancala. The game comes to an end according to the following rules:

(a) A player captures more than 24 seeds to win the game

(b) Both players have equally captured 24 seeds leading to a draw

(c) There are fewer seeds on the board that neither player can ever capture seeds. The player with the highest number of seeds wins the game while equal number of seeds captured by both players connotes a draw.

One important feature of Awale game is that each player has to make a move or select a strategy such that his/her opponent has a legal move to make. If this move does not occur, the opponent will be rewarded with all the remaining seeds on the board and this is referred to as the golden rule (Adewoye, 1990). If during the game, it is discovered that there are not enough seeds to make a capture then both players can advance to a legal move in which they are awarded the seeds in their various holes.

Awale provides strategic thinking and employs some Mathematical and Logical skills that help to teach young people to plan and think ahead. Some scientists do refer to Awale as one of the most arithmetic games that can be used to train children in some counting principles and to build skills. As young and adult players of the game progress during play, they discover strategic importance of planning and the discipline involved in the actual implementation of long-term strategies. They also appreciate the importance of foresight, correct timing and are aware of the principles of cause and effect (Agbinya, 2004). 
International journal on applications of graph theory in wireless ad hoc networks and sensor networks

(GRAPH-HOC) Vol.4, No.2/3, September 2012

\section{RELATED WORK}

There have been some interesting studies on Awale game and one of such studies is due to Olugbara et al. (2007) that attempts to combine minimax search algorithm with Case-Based Reasoning (CBR) to evolve Awale player. In the study, a round-robin tournament was conducted between three players, Minimax, Minimax-CBR and Awale shareware. The average moves, average scores and corresponding standard derivations were recorded to compare the performance of the players. The findings from this study were that combining minimax search with CBR improves the strength of the Awale player, combining minimax search and CBR techniques provides a good result at a shallow search and it provides an efficient heuristic for evolving an Awale player.

Davis and Kendall (2002) presented Awale player with a simple evaluation function to ascertain if co-evolution approach is able to optimize the function to a level where the player can play Awale to a sufficiently high level. Six features were considered for the design of an evaluation function. Daoud et al. (2004) developed Awale player using a genetic algorithm with the objective of showing that a better representation can lead to a deeper search. This particular study added six additional features to those used by Davis and Kendall (2002) to improve the performance of the Awale player and the evolved player was compared against the Awale shareware. The result of this study shows that an evaluation function with more features might not necessary improve the performance of Awale player.

Donkers et al. (2002) gave the formal properties of the Mancala family of games and research opportunities in the fields of mathematics and machine intelligence. The results of the study mainly reflect the mathematical characteristics of Mancala games. Romein and Bal (2002, 2003) constructed an endgame database to solve Awale based on parallel retrograde analysis technique. The result of this study shows that the solution to Awale is draw if both players play optimally. Lincke and Marzetta (2000) constructed a database that contains all board positions with 35 or less seeds. The result of this study shows that to improve the playing strength of Awale, it is better to have a larger endgame database than having a better evaluation function.

Broline and Loeb (1995) provided fundamental incentives to apply combinatorics and endgame considerations to Awale. They analyzed Mancala games with the use of fictitious variant of Mancala called Tchoucallon. This fictitious game is a good example of an endgame that enables a player to immediately capture 20 seeds on the board giving only 1 seed to an opponent. For every move by the opponent, the player makes a move to capture 2 seeds until 1 seed remains on the board for the opponent. However it has not been proven the extent that Tchoucallon can help to suggest moves.

\section{THE HYBRID TECHNIQUE}

The fundamental principle underlying the proposed hybrid technique is to avoid looking into a dataset of Awale strategies for suggesting a move. This can be accomplished by training a machine learning system to predict the outcome of such a move. In this way, Awale player is evolved that fits into the main memory of a computer.

This section will examine pd-clustering and minimum and maximum approximation techniques to evolve an Awale player. First, it is important to discuss minimum and maximum approximation and pd-clustering techniques that we intend to investigate in this study to evolve Awale player.

The Minimum and Maximum (Min/Max) operators approximation technique (Rivest, 1995) 
International journal on applications of graph theory in wireless ad hoc networks and sensor networks

(GRAPH-HOC) Vol.4, No.2/3, September 2012

approximates the minimum or maximum operator with a generalized mean operator. The Min/Max approximation is a special case of the penalty-based heuristic search technique, where the penalties are defined in terms of the derivatives of the approximating function. This approximant is the generalized mean operator, defined as follows:

$$
\mathrm{M}_{\mathrm{p}}(a)=\left(\frac{1}{n} \sum_{i=1}^{n} a_{i}^{p}\right)^{1 / p}
$$

Where $(a)$ is the vector of $(n)$ positive real numbers and $(P)$ is the non-zero real number. To properly understand the concept of Min/Max approximation, it is first important to discuss game tree and Penalty based iterative search. This then set the stage for the discussion of Min/max approximation technique. The game tree begins with a tree $\mathrm{C}$ with root $\mathrm{s}$ and the tree is divided into two subsets, which are either minimum or maximum depending on which player is to play. Moreso, each leaf has an associated value $V(\mathrm{t})$, which can be defined as the value from the terminal position of Max player. To determine the value $\mathrm{v}(\mathrm{c})$ of any configuration, we will induct the structure of the tree and determine $\mathrm{v}(\mathrm{c})$ as:

Based on Equation (2), Max player will select any composition $d \in S(c)$ with maximum value $\mathrm{v}(\mathrm{d})$ and if $\mathrm{c} \in \mathrm{Min}$, player Min will select a composition $d \in S(c)$ with minimum value $\mathrm{v}(\mathrm{d})$ until the game tree reaches its terminal node.

$$
\mathrm{v}(\mathrm{c})= \begin{cases}\mathrm{v}(\mathrm{c}), & \text { if } \mathrm{c} \in \mathrm{T}(\mathrm{C}) \\ \max \mathrm{v}(\mathrm{d}), & \text { if } \mathrm{c} \in \operatorname{Max} \backslash \mathrm{T}(\mathrm{C}) \\ \mathrm{d} \in \mathrm{S}(\mathrm{c}) & \\ \min \mathrm{v}(\mathrm{d}), & \text { if } \mathrm{c} \in \operatorname{Min} \backslash \mathrm{T}(\mathrm{C}) \\ d \in S(c) & \end{cases}
$$

Penalty based iterative search helps to determine which leaf to expand that has the least penalty $(\mathrm{P})$ to get the best result, where $\mathrm{w}$ is the weight and $\mathrm{d}$ is the offspring of $\mathrm{c}$.

$$
\mathrm{P}(\mathrm{c})=\sum_{d \in A(c)} w(d)
$$

Where the weight is determined by

$$
\mathrm{W}(\mathrm{c})=\alpha+\left(\begin{array}{l}
\sim \\
v E(d)-v_{E}(c)
\end{array}\right)^{2}, \quad \text { where } \alpha>0
$$


International journal on applications of graph theory in wireless ad hoc networks and sensor networks

(GRAPH-HOC) Vol.4, No.2/3, September 2012

For large good or bad value of $\mathrm{p}, M_{p}(a)$ which can be a positive approximation for player Max or Min respectively, we will implement the generalized mean which unlike Min/Max

approximation has continuous derivatives with respect to $a_{1}$.The partial derivative of Equation (1) is as follows:

$$
\frac{\partial M_{p}(a)}{\partial a_{i}}=\frac{1}{n}\left(\frac{a_{i}}{M_{p}(a)}\right)^{p-1}
$$

The generalized mean derivative can be taken at each node which can be used in approximating Min/Max functions ,thereby assisting to determine the root in a game tree which a leaf depends on strongly. However, the penalty based iterative search will be employed to determine which node should be expanded as shown in the Equation (6) below:

$$
\tilde{v}_{E}(c)=\left\{\begin{array}{l}
\tilde{v}_{p}(c), \quad \text { if } \quad c \in T(C) \\
M_{p}\left(\begin{array}{c}
\tilde{v}_{E}\left(d_{1}\right), \ldots . ., \tilde{v}_{E}\left(d_{k}\right) \\
M_{-p}\left(\tilde{v}_{E}\left(d_{1}\right), \ldots . ., \tilde{v}_{E}\left(d_{k}\right)\right),
\end{array}\right. \\
\text { if } c \in \operatorname{Max} \backslash T(C)
\end{array}\right\}
$$

The theory on which the PD-clustering is explained below, given data points to be vectors $\mathrm{x}=\left(x_{1}, x_{2}, x_{3}, \ldots x_{n}\right) \in \mathfrak{R}^{n}$ and let a dataset $\mathrm{D}$ consists of $\mathrm{N}$ data points $\left\{x_{1}, x_{2}, x_{3}, \ldots . x_{n}\right\}$.The general problem of data clustering is to partition a dataset into $\mathrm{m}$ clusters of similar data points. The pd-clustering technique relates probability and distance using a simple inverse principle. For each $x \in D$ and cluster centroid $c_{k}$, the probability $p_{k}(x)$ that $x$ belongs to $\mathrm{D}$ is given as

$$
\frac{p_{k}(x) d_{k}(x)}{q_{k}}=\text { Cons } \tan t
$$

This result can be interpreted as meaning that cluster membership is more probable the closer the data point is to the cluster centroid and the larger the cluster. Iyigun and Ben-Isreal (2008) have shown that Equation (7) is the solution of the following extremal problem: 
International journal on applications of graph theory in wireless ad hoc networks and sensor networks

(GRAPH-HOC) Vol.4, No.2/3, September 2012

$\min \left\{\sum_{\mathrm{i}=1}^{\mathrm{N}}\left(\frac{d_{1}(x) p_{1}^{2}(x)}{q_{1}}+\frac{d_{2}(x) p_{2}^{2}(x)}{q_{2}}\right) \| p_{1}(x)+p_{2}(x)=1, p_{1}(x), p_{2}(x) \geq 0\right\}$

Where $\mathrm{d}_{1}(\mathrm{x})$ and $\mathrm{d}_{2}(\mathrm{x})$ are distances of the data point $\mathrm{x}$ to the cluster of size $\mathrm{q}_{1}$ and $\mathrm{q}_{2}$ and $\mathrm{p}_{1}(\mathrm{x})$ and $\mathrm{p}_{2}(\mathrm{x})$ are the cluster probabilities. To solve Equation (8), the Lagrangian of the problem is defined as:

$\mathrm{L}\left(\mathrm{p}_{1}(x), p_{2}(x), \lambda\right)=\sum_{i=1}^{N}\left(\frac{d_{1}(x) P_{1}^{2}(x)}{q_{1}}+\frac{d_{2}(x) P_{2}^{2}(x)}{q_{2}}\right)-\lambda\left(p_{1}(x)+p_{2}(x)-N\right)$

By zeroing the partial derivatives $\frac{\partial L}{\partial P_{i}}$ gives the solution to Equation (9) as follows:

$$
\mathrm{P}_{\mathrm{k}}(x)=\frac{\prod_{j \neq k} d_{j}(x) / q_{j}}{\sum_{i=1}^{K} \prod_{j \neq i} d_{j}(x) / q_{j}}
$$

where $\mathrm{K}$ is the number of clusters. The distance function $\mathrm{d}(\mathrm{x}, \mathrm{y})$ that measures the closeness of the vectors $\mathrm{x}$ and $\mathrm{y}$ is usually given as:

$$
d(x, y)=\|x-y\|, \forall \mathrm{x}, \mathrm{y} \in \mathfrak{R}^{\mathrm{n}}
$$

Where II.II is a norm. There are several norms for distance computation and examples include Chebycheu, Proscrute, Euclidean and Mahalanobis, which is preferred than the Euclidean because it is consistent across conditions and it pays equal attention to all components.

$$
\mathrm{d}\left(\mathrm{x}, \mathrm{c}_{\mathrm{k}}\right)=\left\{\left(x-c_{k}\right)^{T} \sum_{k}^{-1}\left(x-c_{k}\right)\right\}^{1 / 2}
$$

Where $\mathrm{A}^{\mathrm{T}}$ means transpose vector of $\mathrm{A}$ and $\sum_{\mathrm{k}}^{-1}$ is the inverse matrix of the covariance matrix $\sum_{\mathrm{k}}$ given by

$$
\sum_{k}=\frac{\sum_{i}^{N} u_{k}\left(x_{i}\right)\left(x_{i}-c_{k}\right)\left(x_{i}-c_{k}\right)^{T}}{\sum_{i=1}^{N} u_{k}\left(x_{i}\right)}
$$

Where

$$
\mathrm{u}_{\mathrm{k}}\left(x_{i}\right)=\left[\left(\frac{\mathrm{d}_{1}\left(x_{i}, c_{1}\right)}{q_{1}}\right)^{2} \frac{1}{\mathrm{~d}_{2}\left(x_{i}, c_{2}\right)}\right]\left(\frac{\mathrm{d}_{1}\left(x_{i}, c_{1}\right)}{q_{1}}+\frac{\mathrm{d}_{2}\left(x_{i}, c_{2}\right)}{q_{2}}\right)^{-2}
$$


International journal on applications of graph theory in wireless ad hoc networks and sensor networks

(GRAPH-HOC) Vol.4, No.2/3, September 2012

\section{IMPLEMENTATION OF HYBRID TECHNIQUE}

The algorithm is designed in such a way that the moves (strategies) are classified into 2 classes $c_{1}$ and $c_{2}$ which represent good and bad strategies respectively. Using our evolved hybrid pseudocode which assists to finds the mahalanobis distance for each strategy on the current board state for both classes of strategies, of which the result of the bad strategy is divided by the sum of both the good and bad strategies. Thereby selecting the highest possible score as the best.The algorithm is described more compactly by the following pseudo-code:

(1) Given a game state, let the vector move $[s]=\left\{m_{1}, m_{2}, \ldots, m_{k}\right\}$ be a set of $\mathrm{S}$ feasible moves. Where there can be $s_{1} \ldots s_{6}$ number of possible strategies

(2) Classify the moves into $c_{1}$ and $c_{2}$ classes using Tchoucallion (strategies)

(3) If $c_{1}$ and $c_{2}$ are not empty matrixes, then find the inverse (covariance) of the respective matrices which are ${ }^{I_{g}}$ and $I_{b}$, where $c_{1}$ is the good strategies $c_{2}$ while are the bad strategies.

(4) Let $M_{g}=\left\|c_{1}\right\|_{\text {and }} M_{b}=\left\|c_{2}\right\|_{\text {where }} M_{g}$ and $M_{b}$ are the respective means of good and bad strategies.

(5) If $c_{g}=1$ which as a determinant (mean) of ${ }^{c_{g}}$ of good strategies which satisfies equation5below:

$D_{g}=\sqrt{\left(\left(s-M_{g}\right) * I_{g} *\left(s-M_{g}\right)^{T}\right)}$

Else select $c_{b}=0$ to satisfy equation

$D_{b}=\sqrt{\left(\left(s-M_{b}\right) * I_{b} *\left(s-M_{b}\right)^{T}\right)}$

(6) Compute the Distance

$$
(D)=\frac{D_{b}}{D_{b}+D_{g}}
$$

bad and good strategies

(7) Select the highest of the possible strategies as the best strategy using this equation for all available strategies

\section{RESULTS}

To evaluate the performance of our evolved player, the player played a series of games with Awale in tournaments. First, we registered for the Awale program before a direct access is given to play at all levels, but the initial level is free. The results obtained from the series of 10 games at each level of the game (each player starts 5 times) played are recorded in the table below.

We record moves up to the level where a player has captured the maximum number of seeds required to win or draw. However, in practice the game continues until fewer seeds (say three) remain on board.The four levels are;

(1) Playing PDC-Minimax against Minimax

(2) Playing PDC-Minimax against Awale at Initiation level

(3) Playing PDC-Minimax against Awale at Beginner Level

(4) Playing PDC-Minimax against Awale at Amateur level

(5) Playing PDC-Minimax against Awale at Grandmaster level 
International journal on applications of graph theory in wireless ad hoc networks and sensor networks (GRAPH-HOC) Vol.4, No.2/3, September 2012

Table 1. The outcome of the experiment.

\begin{tabular}{|c|c|c|}
\hline PDC-Minimax(Average) & Minimax(Average) & No of Moves \\
\hline 28.00 & 7.00 & 38.50 \\
\hline PDC-Minimax & Awale(initiation level) & No of Moves \\
\hline 26.00 & 5 & 23 \\
\hline PDC-Minimax & Awale(beginner level) & No of Moves \\
\hline 26.00 & 7.6 & 33 \\
\hline PDC-Minimax & Awale(Amateur level) & No of Moves \\
\hline 39.6 & 18.6 & 39.6 \\
\hline PDC -Minimax & Awale(Grandmaster level) & No of Moves \\
\hline 61.6 & 25 & 61.6 \\
\hline
\end{tabular}

The result in Table 2 shows that Minimax-PDC performed very well against all the opponents except at the grandmaster where it competed vigorously but lost the game at the end. The combination of Minimax and PDC has shown that they can be combined to reasonably play Awale.

\section{CONCLUSION}

A heuristic is presented that combines minimax search and PDC to evolve an Awale agent. The heuristic was tested by training an aggregate malanobis distance reasoned with strategies acquired from human agent. However, it would be interesting to investigate more extensively the PDC training with extracts of the endgame database by[6], since the Awale game is similar to Ayo, and Awale is solved using the endgame database. The results of the experiment performed shows that combining minimax search with PDC improves the playing strength of the Awale agent, it provides a good result at a deeper search, and give an efficient heuristic for evolving an Awale agent.

\section{REFERENCES}

[1] Adewoye, T. O. (1990). On Certain Combinatorial Number Theoretic Aspects of the African Game of 'Ayo'. AMSE Review, Vol. 14, No. 2, pp. 41- 63.

[2] Agbinya, J.I (2004). Computer Board Games of Africa (Algorithm, Strategies and Rules), Available at http://services.eng.uts.edu.au/agbinya/computer\%20games/African Board Games.pdf. Accessed on 23/10/2010.

[3] Allis, V., Muellen, M.V.D and Herik, J.V.D. (1994). Proof-Number Search, Artificial Intelligence, Vol .66, No 1. pp 91-124.

[4] Akinyemi, I. O., Olugbara, O.O., Longe, H.O.D. and Adebiyi, E.F. (2008). On Nearest Neighbour Strategy for Evolving Ayo Game Player. Journal of Computer Science and its Application (An International Journal of the Nigeria Computer Society) Vol. 15, pp. 48 - 58. 
International journal on applications of graph theory in wireless ad hoc networks and sensor networks (GRAPH-HOC) Vol.4, No.2/3, September 2012

[5] Akinyemi, I. O , Adebiyi, E. F and Longe, H.O.D (2009). Critical Analysis of Decision Making Experience with a machine Learning Approach in Playing Ayo Game. World Academy of Science, Engineering and Technology.(WASET),56,49-54.

[6] Broline, D.M. and Loeb, D.E. (1995). The Combinatorics of Mancala-type games: "Ayo", Tchoukaillon and UMAP J., Vol .1, Nos., 41, pp.1-15.

[7] Davis, J.E. and Kendall, G. (2002). An Investigation, using Co-evolution, to Evolve an Awari Player. Proceedings of Congress on Evolutionary Computation (CEC), pp. 1408-1413.

[8] Daoud M., Kharma, N., Haidar, A. and Popoola, J. (2004). Ayo the Awari Player, or How Better Representation Trumps Deeper Search, Proceedings of the 2004 IEEE Congress on Evolutionary Computation, pp. 1001-1006.

[9] Donkers, H.H.L.M., Uiterwijk, J.W.H.M.and Voogt, A.J.De (2002). Mancala Games Topics in Artificial Intelligence and Mathematics. Step by Step Proceedings of the 4th Colloquium 'Board Games in Academia' (eds. J. Retschitzki and R. Haddad-Zubel), Editions Universitaires, Fribourg,Switserland. pp. 133-146

[10] Iyegun, C. and Ben-Isreal, A. (2008). Probabilistic Distance Clustering Adjusted for Cluster Size. Probability in the Engineering and Informational Sciences, 22, 603-621.

[11] Lincke, T. R., \& Marzetta, A. (2000). Large endgame databases with limited memory space. International Computer Games Association Journal, Vol.23, No 3, pp 131-138.ISSN 0920-234X.

[12] Olugbara, O.O., Adigun, M.O., Ojo, S.O and Adewoye, T.O. (2007). An Efficient Heuristic for Evolving an Agent in the Strategy game of Ayo. International Computer Games Association Journal, Vol. 30, pp 92-96.

[13] Olugbara, O.O., Adewoye, T.O and Akinyemi, I.O. (2006). An Investigation of Minimax Search Techniques for Evolving Ayo/Awari Player. Proceedings of IEEE-ICICT 4th International Conference on Information and Communication Technology, Cairo, Egypt, pp. 1-1.

[14] Rivest, L.R. (1995). Game Tree Searching by Min/Max Approximation. MIT Laboratory for Computer Science. Cambridge, Massachusetts, USA., pp.,1-22. Available at: http://people.csail.mit.edu/rivest/Rivest-GameTreeSearchingByMinMaxApproximation.pdf. Accessed: 22/01/2011.

[15] Romein, J.W. and Bal, H.C. (2002). Awari is solved. International Computer Games Association Journal, Vol. 25, Nos. 3. pp. 162-165.

[16] Romein, J.W. and Bal, H.C. (2003). Solving the game of Awari using Parallel Retrograde Analysis. In Proceedings of IEEE Computer Society, Los Angeles, USA., Vol. 36, Nos.10. pp. 23-33.

\section{Authors}

Keneilwe Zuva is currently a lecturer at the University of Botswana in the Department Of Computer Science. She received Masters of Engineering in Information Systems and Networking from the University of Essex, UK in 2001. Her research interests are networking, image processing, and network security.

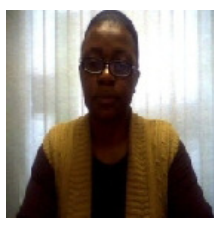

